\section{Bedside neurology}

Categorising the causes of unilateral paralysis of the lower cranial nerves is almost as popular a clinical exercise as defining the causes of wasting of the small muscles of the hand. Cerebrovascular accidents are unlikely to be high on most clinicians' lists, for the lesions that come to mind are infiltration of the meninges by metastatic carcinoma or sarcoid, or compression of nerves by glomus jugulare and other tumours in the region of the jugular foramen, or intrinsic lesions of the brain stem such as syringomyelia or gliomas-in short, lower motor neurone lesions. The more discerning might suggest that strokes affecting the cerebral hemispheres and other upper motor neurone lesions should be associated with movements of the opposite side of the body rather than with individual muscles.' Furthermore, many of the functions of the cranial nerves have bilateral innervation and may be little affected by a unilateral stroke. As a result-apart perhaps from the well known distinction between the two types of facial paralysis in upper and lower motor neurone lesions-little attention is paid to dysfunction of the lower cranial nerves in patients who have had a stroke.

In these days of "scan negative headache" 2 it is particularly refreshing to read an account of the careful clinical investigation of the function of the lower cranial nerves in patients who have sustained a stroke (p 791). The findings, backed by but not oppressed by statistical analysis, confirm much of current clinical thought, but in addition bring out several interesting points. For example, to take the fifth cranial nerve, the jaw muscles are thought to have bilateral innervation, and when the pterygoids are the only muscles tested this might seem to be the case, but in patients with stroke the masseters may show definite unilateral weakness (provided that precautions are taken to avoid possible confusion caused by facial paralysis and loss of teeth). Every student is taught that a lower motor neurone lesion of the facial nerve results in paralysis of both upper and lower parts of the face on the same side as the lesion, and an upper motor neurone lesion only the lower part of the face on the opposite side. If the stroke is severe or acute, however, there may also be weakness of the upper part of the face, though this is not as definite or as long lasting as the lower facial weakness. The authors found this weakness in about one quarter of their patients, and, though they correlate it with the severity of the hemiplegia, they do not emphasise the importance of examining for weakness soon after the acute episode. Sometimes the upper face is affected severely and the picture may mimic a lower motor neurone lesion. ${ }^{3}$ The vital distinction here is that the accompanying hemiplegia will be on the same side, whereas if the lesion was in the brain stem it would be on the opposite side. This often causes confusion in practice.

The observations on the function of the sternomastoids are particularly interesting. Using the test of turning the head against resistance, which brings into action the muscle opposite to the side to which the head is turning, Willoughby et al have shown that after a stroke it is the ability to turn the head towards the hemiparetic side that is affected, not the action of the muscle contralateral to the lesion..$^{1+5}$ They do not record, however, whether, if the head was flexed forwards against resistance, bringing both sternomastoids into action simultaneously, the appropriate muscle was found to be weakened. This would make an interesting study because in practice it does not appear to be the case. We have to remember that other neck muscles play a part in the performance of these tests.

Clinical life would be easy if rules were always obeyed, but the behaviour of the twelfth nerve seems to be an exception. After a stroke it is uncommon to find weakness on one side of the tongue but when there is it would generally be expected to be on the side of the hemiplegia, and this observation was confirmed in this recent study. But, taking up the principle of movement rather than muscle-and remembering that the genioglossus pushes the tongue forwards and to the opposite side-we might expect that a unilaterally innervated tongue from supranuclear pathways would be unable to push the tongue towards the side of the hemiplegia, whereas the opposite is the case; this has not been examined critically by most authors.

Another interesting side issue from the present study relates to speech disorders. If a patient has true dysphasia he or she has a lesion in the dominant hemisphere. Dysarthria, however, may be caused by lesions at any site in the pathways concerned in the mechanics of speaking; it may occur in patients who do not have dysphasia or weakness of the tongue when the lesion is in one cerebral hemisphere, and not necessarily the dominant one. The presence of dysarthria without dysphasia need not lead one to try to locate a vascular lesion in the brain stem or cerebellumsomething which clinicians may tie themselves up in knots trying to do.

Working out the site of a lesion from the history and clinical examination should (even in the most well equipped departments) be the linchpin of bedside teaching for both undergraduates and postgraduates. It is good to see this paper coming from a group of young physicians.

\section{EDWIN R BICKERSTAFF}

Honorary Consulting Neurologist,

Midland Centre for Neurosurgery and Neurology,

Smethwick,

West Midlands

1 Reeves AG, Valenstein E, Ochoa JL, Woodford JE. Disorders of the nervous system. A primer. Chicago: Year Book Medical Publishers Inc, 1981.

2 Marshall J. Different types of neurologist. Br Med f 1984;288: 1994

Bickerstaff ER. Neurological examination in clinical practice. 4th ed. Oxford: Blackwell Scientific Publications, 1980

4 Balagura S, Katx RG. Undecussated innervation to the sternocleidomastoid muscle: a reinstatement. Ann Neurol 1980;7:84-5.

the decussated innervation of the sternocleidomastoid muscle. Ann Neurol 1981;10:495.

\section{Correction}

Ample scope for improving the health of prisoners

Dr Richard Smith referred in a leading article (30 June, p 1939) to concern about "frequent newspaper reports of medical mishaps in our overcrowded and thoroughly unpleasant prisons." Reference was made to an article in The Times about the suicide of David Martin in which an "anonymous psychiatrist" was said to have "warned the prison authorities that the newly convicted David Martin was a high suicide risk just before he hanged himself in Parkhurst prison.'

The medical staff at Parkhurst point out that no such warning was given "just before" Mr Martin hanged himself, nor did The Times say that it was. Secondly, the staff had access to all the available material concerning Mr Martin's psychiatric state before he was transferred to Parkhurst prison and they acted on this coupled with their own clinical assessment; and there was no question of any criticism of the medical officers being in anyway justified.

We are glad to apologise if the impression was given that the medical staff were at fault. They for their part accept that Dr Smith's intention in referring to Mr Martin's case in conjunction with another case was merely to give examples of cases which had attracted newspaper reports. 